\title{
Cementoblastoma de maxila: Reporte de caso de un tumor odontogénico raro y breve revisión de la literatura
}

Cementoblastoma of maxilla: Case report of a rare odontogenic tumor and short review of the literature.

\author{
Wilson Delgado Azañero ${ }^{1, a}$, Emylu Miranda Castillo ${ }^{2, b}$, Victor Calderon Ubaqui ${ }^{1, c}$, \\ Grover Cordero Peña ${ }^{1, d}$
}

\section{RESUMEN}

El cementoblastoma es un tumor benigno de origen odontogénico ectomesenquimal que ocurre muy raramente en la maxila. Se presenta el caso de una paciente femenina de 22 años de edad, con asimetría facial producida por un tumor duro levemente doloroso que expandía las corticales óseas vestibular y palatina a nivel de la segunda premolar superior derecha. Se discute las dificultades del diagnóstico y el aporte de los estudios imagenológicos con TC de haz cónico (TCHC). El tumor fue removido en bloque con la premolar involucrada y los dos dientes contiguos. Se presentan las características histopatológicas del cementoblastoma demostrando que se produce reabsorción por reemplazo en la raíz del diente comprometido.

PALABRAS CLAVE: Cementoblastoma, tumores odontogenicos, maxila.

\section{SUMMARY}

Cementoblastoma is a benign tumor derived from the ectomesenchymal odontogenic tissues. Their occurrence in the maxilla is extremely rare. A case of a 22-year-old female patient is presented. Main complain was facial asymmetry produced by a hard tumor that affected the first upper right premolar tooth which expanded vestibular and palatine cortical bone. Mild pain, particularly when touching was manifested by patient. Difficulties to establishes the correct diagnosis and the contribution of cone beam CT (CBCT) are discussed. Surgical block resection that included the premolar together with contiguous teeth was the treatment. The microscopic features of cementoblastoma are shown demonstrating substitution resorption of affected tooth root.

KEY WORDS: Cementoblastoma, odontogenic tumors, maxilla. 


\section{INTRODUCIÓN}

El cementoblastoma es un tumor benigno originado en el ectomesénquima odontogénico; según Dogra et al., fue reconocido por primera vez por Dewey en 1927(1), sin embargo, para Bal et al., fue Norberg en 1930 quien realizó la primera descripción de esta lesión (2). La OMS define a esta entidad como un tumor formado por un tejido mineralizado parecido al cemento fusionado a la raíz de un diente, macroscópicamente consiste de una masa dura redonda o nodular adherida a uno o más raíces de un diente rodeada por un tejido blando blanco grisáceo (3).

Clínicamente se presenta como un aumento de volumen doloroso alrededor de la raíz de un diente que produce una deformación de la zona vestibular y/o lingual o palatina, generalmente el diente involucrado conserva su vitalidad. Aparte del dolor otros síntomas neurológicos no se han reportado. Radiográficamente aparece como una masa radiopaca o de densidad mixta fusionada con la raíz de un diente, rodeada por un espacio delgado radiolúcido que envuelve completamente a la lesión.

Histológicamente se observan masas densas de material parecido a cemento dentro de un estroma de tejido conectivo laxo vascularizado donde se distinguen cementoblastos y cementoclastos multinucleados. La masa calcificada tumoral se continúa con la raíz del diente afectado produciendo reabsorción. En las partes más maduras de la lesión existen líneas de reversión basofílicas que producen un patrón óseo parecido a los cambios observados en la enfermedad de Paget (3). En la periferia de la lesión se observan columnas de material calcificado distribuidas en forma radial, el estroma intercolumnar se continúa con el tejido fibroso que rodea a la lesión.

El cementoblastoma, es considerado como la única neoplasia del cemento dental, por otro lado, es una lesión muy rara que representa menos del $1 \%$ de los tumores odontogénicos. Aproximadamente el $50 \%$ se diagnostica en pacientes menores de 20 años y el $75 \%$ en pacientes debajo de los 30 años, aparentemente no hay predilección por sexo (1).

Se desarrolla predominantemente en la mandíbula, la localización más frecuente corresponde a molares y premolares mandibulares permanentes siendo la primera molar la más afectada $(4,5)$. En maxilar superior son raros, afecta también la zona molar y premolar, en algunos casos el seno maxilar puede estar comprometido (6), existe el reporte de un cementoblastoma adherido a una tercera molar superior impactada (7). En la población pediátrica los cementoblastomas son muy raros, según la literatura hasta el 2019 solo se habían reportado 17 casos $(8,9,10)$. Es notorio el caso de un niño que en un periodo de 5 años desarrolló tres cementoblastomas mandibulares, uno de ellos se inició como una elongación radiolúcida de la raíz mesial de la pieza 46 (11).

En la literatura existen dos trabajos importantes que han estudiado grandes series de casos de cementoblastomas con la finalidad de analizar sus características clínicas, radiográficas tratamientos $\mathrm{y}$ recurrencia cuyos hallazgos difieren en algunos aspectos. Uno de ellos analizó 44 casos (12), y el otro 258 casos (13). La mayor parte de los resultados coincidían con lo reportado en la literatura, sin embargo, en el primer estudio (12), se encontraron dos casos que radiográficamente aparecieron como imágenes radiolúcidas y en el seguimiento de toda la muestra a los 5,5 años se encontró una recurrencia de $37,5 \%$. En el segundo trabajo (13), 77,5\% de cementoblastomas estuvieron en la mandíbula, el $70,2 \%$ presentó sintomatología dolorosa, el $78 \%$ tenía el diente vital, el $98,2 \%$ se presentó como imagen radiopaca, en el 59,8 \% hubo reabsorción radicular, el 23,6\% produjo desplazamiento del canal mandibular, en el 16,3\%, se halló perforación de la tabla cortical, el 13,4\% produjo desplazamiento de dientes, y en el control de 30,6 meses la recurrencia fue de $11,8 \%$.

En el diagnóstico diferencial se consideran lesiones que producen radiopacidades tales como: osteítis condensante focal, osteoblastoma, osteoma central, displasia cemento ósea focal, odontoma complejo, hipercementosis (1-6). En relación con malignidad los cementoblastomas pueden ser confundido con osteosarcoma sobre todo por la imagen radiográfica que producen de la misma forma osteosarcomas pueden ser confundido por cementoblastomas $(14,15)$.

El tratamiento del cementoblastoma depende de la ubicación y de su tamaño, puede ser cirugía 
conservadora, es decir extracción del diente involucrado conjuntamente con el tumor, o puede realizarse resección marginal o resección segmentaria. En algunos casos erróneamente sólo se extrae el diente dejando la lesión la cual posteriormente puede ser identificada radiográficamente como una masa radiopaca en el sitio de la extracción, estos casos se consideran cementoblastomas residuales (16).

El propósito de este trabajo es reportar las características clínicas, radiográficas, histopatológicas y el tratamiento de un cementoblastoma asociado

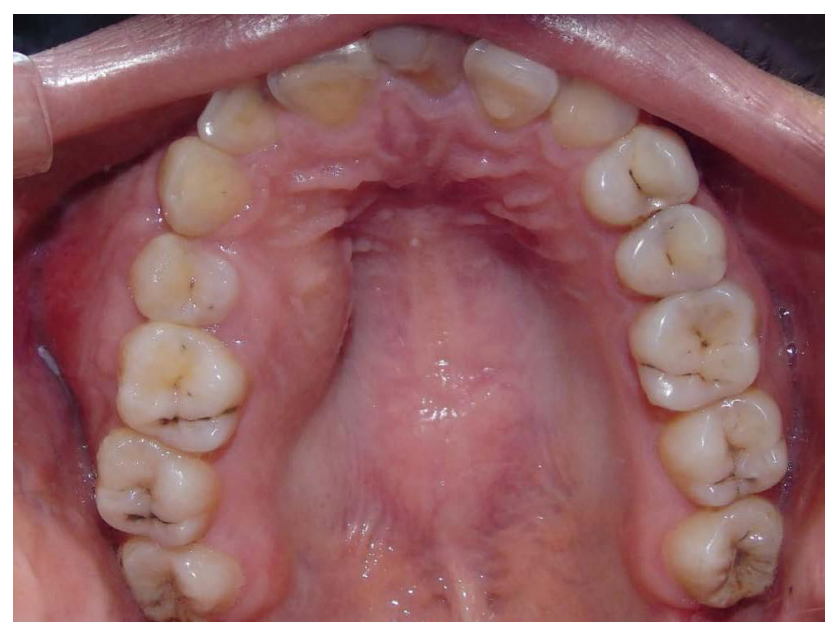

Figura 1. Tumor que expande la tabla ósea vestibular y palatina a nivel pieza 15 y 16 . Mucosa oral intacta. a una primer premolar superior, discutir las dificultades surgidas para su diagnóstico y presentar la contribución de la Tomografía computarizada de haz cónico (TCHC) en el estudio imagenológico de la lesión.

\section{Reporte de caso}

Paciente femenina de 22 años de edad, natural y proveniente de la ciudad de Iquitos, quien acude al servicio de Medicina y Cirugía oral y Maxilofacial de la Facultad de Estomatología de la Universidad Peruana Cayetano Heredia por presentar un tumor en el maxilar superior del lado derecho, de un año de duración, de crecimiento progresivo acompañado de sintomatología dolorosa moderada que no le impedía alimentarse ni hablar. En la historia médica no se encontraron antecedentes mórbidos, durante el examen físico no se identificaron adenopatías cervicales.

El examen extraoral reveló asimetría facial debida a un abultamiento de consistencia dura y dolorosa que deformaba la fosa canina derecha. El examen intraoral mostraba un tumor de forma redonda que comprometía la zona vestibular y palatina de la pieza 15 y parte de la encía de la pieza 16, había desaparición del fondo del surco vestibular, a la palpación el tumor tenía consistencia dura y la paciente manifestaba
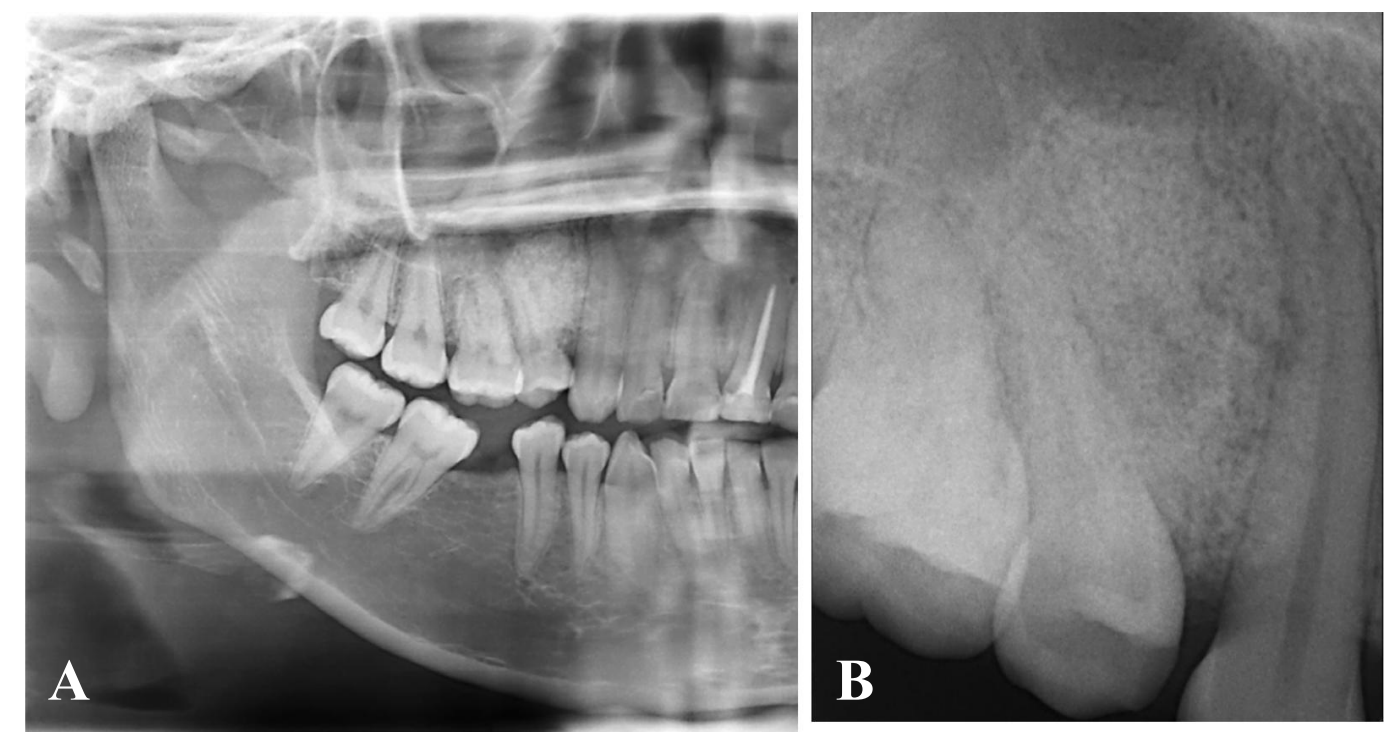

Figura 2. 2a) Radiografía panorámica: muestra en mesial de pieza 15 radiopacidad en forma de pera invertida que se extiende desde la cresta ósea alveolar hasta el límite con el piso del seno maxilar.2b) La radiografía periapical muestra la misma lesión radiopaca con aspecto granular rodeada parcialmente por una línea radiolúcida. 

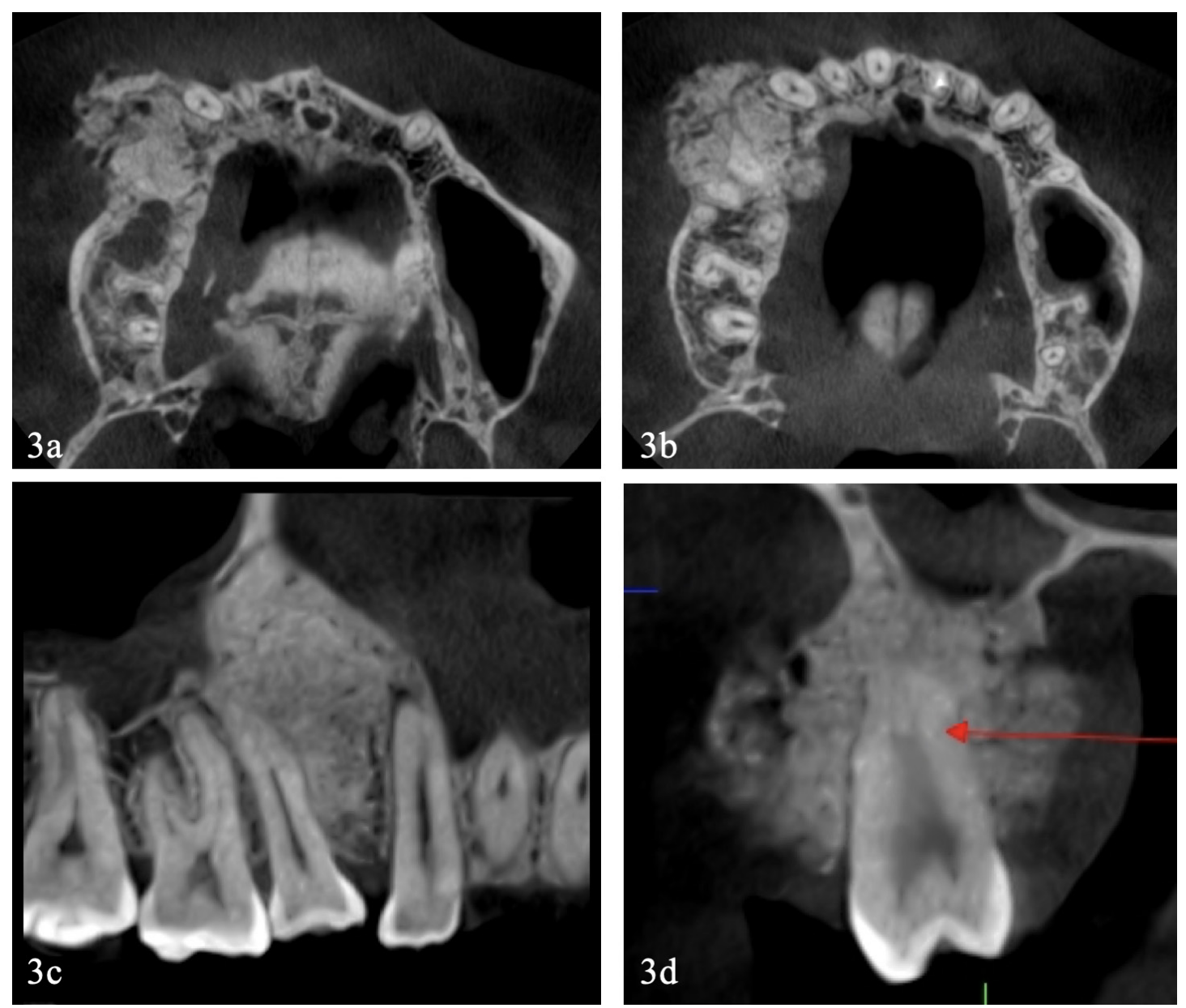

Figura 3. TCHC 3a) C. axial a nivel apical de dientes: masa ubicada entre 13 y 16, forma redonda, hiperdensa con focos isodensos, protruye hacia vestibular. $3 b$ ) C. axial a nivel medio de las raíces: masa hiperdensa heterogénea de bordes regulares que protruía hacia vestibular y palatino.3c) C. sagital: masa hiperdensa, forma de pera invertida, en mesial de 15, separada de la cortical del piso del seno por una línea isodensa.3d) C. transaxial a nivel de 15: masa hiperdensa en vestibular y palatino, el borde vestibular es marcadamente irregular. Leve reabsorción apical (flecha).

dolor de intensidad leve, el tamaño aproximado era de $3 \times 2,5 \times 2 \mathrm{~cm}$, la mucosa que recubría la lesión lucia normal (figura 1). Se notó la ausencia de la pieza 14, las pruebas de vitalidad pulpar de las piezas 13,15 y 16 resultaron normales. Las coronas de los dientes adyacentes estaban conservadas en forma y tamaño y no existía movilidad dental, la pieza 15 estaba ligeramente sumergida, pero la oclusión estaba conservada.

La radiografía panorámica y periapical mostraban un imagen radiopaca de aspecto granular, con forma de pera invertida, localizada entre la pieza 13 y 15 , que se extendía desde la cresta alveolar hasta el piso del seno maxilar, en este nivel, bordeando parcialmente la lesión se notaba una línea radiolúcida no bien definida, no había evidencia de reabsorción radicular (figura 2 (a y b).Con los hallazgos clínicos y radiográficos convencionales se plantearon los siguientes diagnósticos diferenciales: osteoma central, osteoblastoma, cementoblastoma, displasia fibrosa y odontoma complejo.

La TCHC proporcionó mayor información sobre las características imagenológicas de la lesión. En el corte axial de la maxila a nivel apical de las piezas de primer cuadrante, se observaba una imagen hiperdensa heterogénea con focos isodensos de forma ovoide y contornos irregulares que ocupaba el centro del reborde alveolar y se proyectaba hacia vestibular (figura 3 (a)). El corte axial a nivel de la parte media de las raíces de los dientes (figura 3 (b)) mostró una masa hiperdensa heterogénea de bordes regulares ubicada de distal de la pieza 13 a mesial de 16 que 

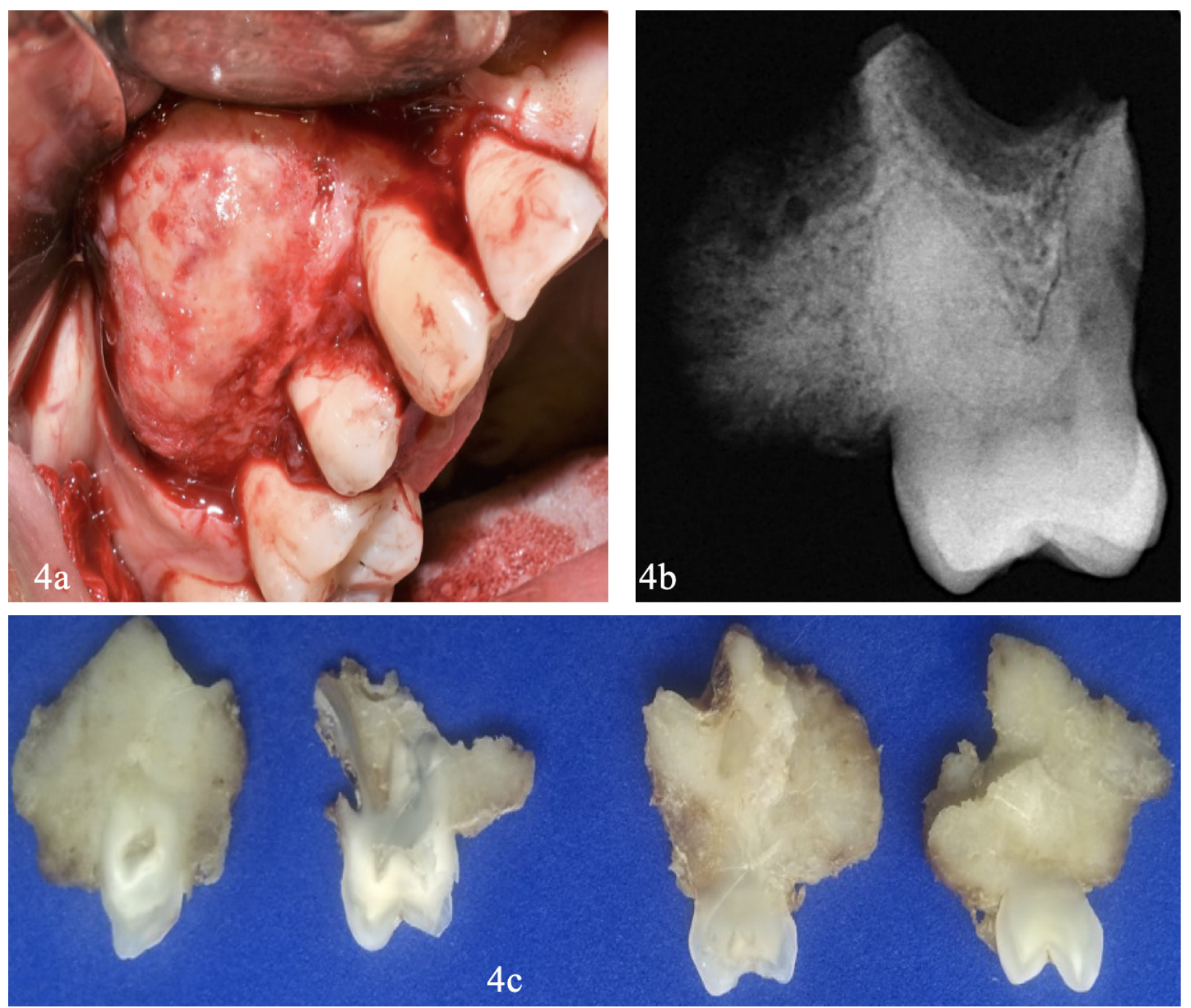

Figura 4. 4a) Imagen transoperatoria: expansión del hueso cortical vestibular en piezas 13,15 16. 4b) Radiografía de la pieza quirúrgica; masa radiopaca adherida a 15 que exhibe proyecciones filamentosas dispuestas radialmente. 4c) Cortes de la pieza quirúrgica a diferentes niveles que muestran una masa grisácea adherida en vestibular y palatino de 15.

protruía hacia vestibular y palatino, su mayor tamaño estaba en vestibular. Comparativamente la densidad de la lesión era menor al de la cortical ósea y a la de la dentina (figura 2 (a y b)).

En el corte sagital se observaba una lesión hiperdensa con forma de pera invertida ubicada en distal de la pieza 13 y mesial de la pieza 15 , se extendía desde la cresta alveolar hasta el piso del seno maxilar, en este nivel se notaba un espacio delgado isodenso que bordeaba a la lesión (figura 3 (c)). El corte transaxial mostraba un tumor de forma más o menos redonda hiperdensa con escaso focos isodensos adherido a la cara palatina y vestibular de la raíz de la pieza 15, en vestibular el contorno era marcadamente irregular debido a pequeñas proyecciones hiperdensas, de apariencia radial. Debido a los hallazgos tomográficos se decidió tomar una biopsia representativa de la lesión, cuyo resultado anatomopatológico fue cementoblastoma. Considerando el diagnóstico de cementoblastoma de pieza 15, con extensión hacia los dientes 13 y 16 , el tratamiento quirúrgico consistió en la remoción en bloque del tumor incluyendo los dos dientes contiguos, siguiendo la técnica de maxilectomía parcial descrito como tipo II B por Brown et al., (17). Durante el acto quirúrgico se comprobó que el tumor expandía marcadamente la cortical vestibular 

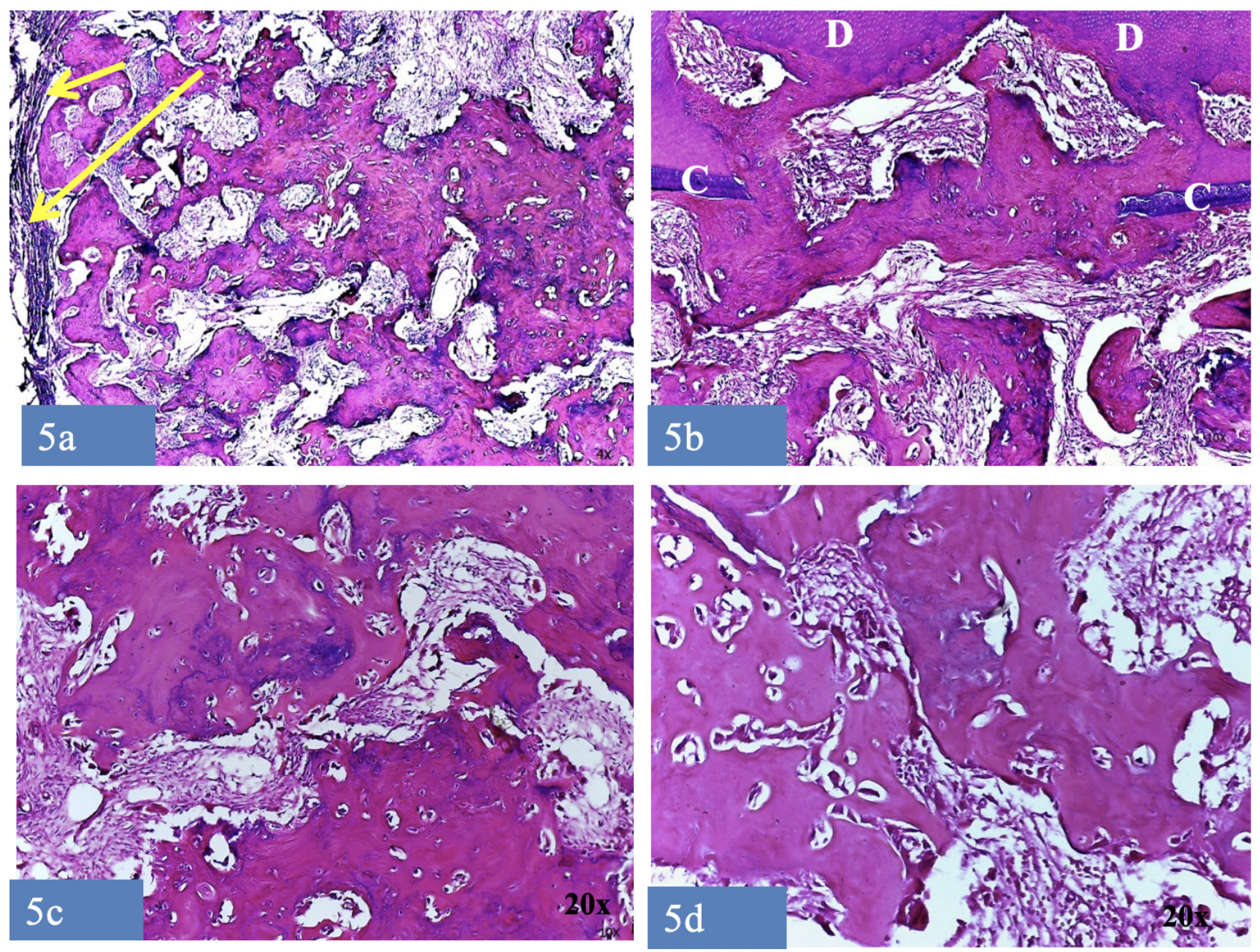

Figura 5. Histopatología: 5a) Cápsula de tejidos conectivo fibroso (flechas) que se continua con el estroma del tumor 5b) Zona de reabsorción radicular por reemplazo: el tumor atraviesa el cemento del diente (C) llegando hasta la dentina (D) la cual está reabsorbida y unida al tumor. 5c) Trabéculas gruesas de cemento con algunas líneas de reversión y estroma de tejido conectivo laxo vascularizado conteniendo cementoblastos y cementoclastos. 5d) En el estroma se distinguen cementoblastos grandes adosados a las trabéculas de cemento.

comprometiendo la parte distal de la pieza13 y mesial de la pieza 16 (figura 4 (a)).

La radiografía del cementoblastoma extirpado revelaba una masa radiopaca fusionada a la raíz de la premolar, cuya periferia mostraba filamentos radiopacos como rayos de sol (figura 4 (b)). El examen macroscópico de cortes realizados a lo largo del espécimen quirúrgico mostraba un tumor compuesto por una masa calcificada de color blanco grisáceo adherida por vestibular y palatino a la raíz de la premolar afectada (figura 4 (c)).

En el examen histológico de cortes representativos del tumor identificaron las características del cementoblastoma. La figura 5 (a) muestra la cápsula de tejido fibroso que envuelve al tumor, el cual se continúa con el estroma presente entre las trabéculas de cemento desorganizado. La figura 5 (b) muestra la zona de reabsorción por reemplazo o sustitución a nivel de la dentina radicular, se ve que el tumor ha perforado el cemento normal del diente y se fusiona con la dentina reabsorbida, La figura 5 (c y d) muestra trabéculas gruesas de contornos irregulares formadas por cemento desorganizado con un estroma de tejido conectivo laxo vascularizado que contiene cementoblastos voluminosos adosados a las trabéculas de cemento y algunos cementoclastos. No se distinguían mitosis. El control clínico y radiográfico a los 8 meses no evidenciaba recurrencia del tumor (figura 6 (a y b)). 

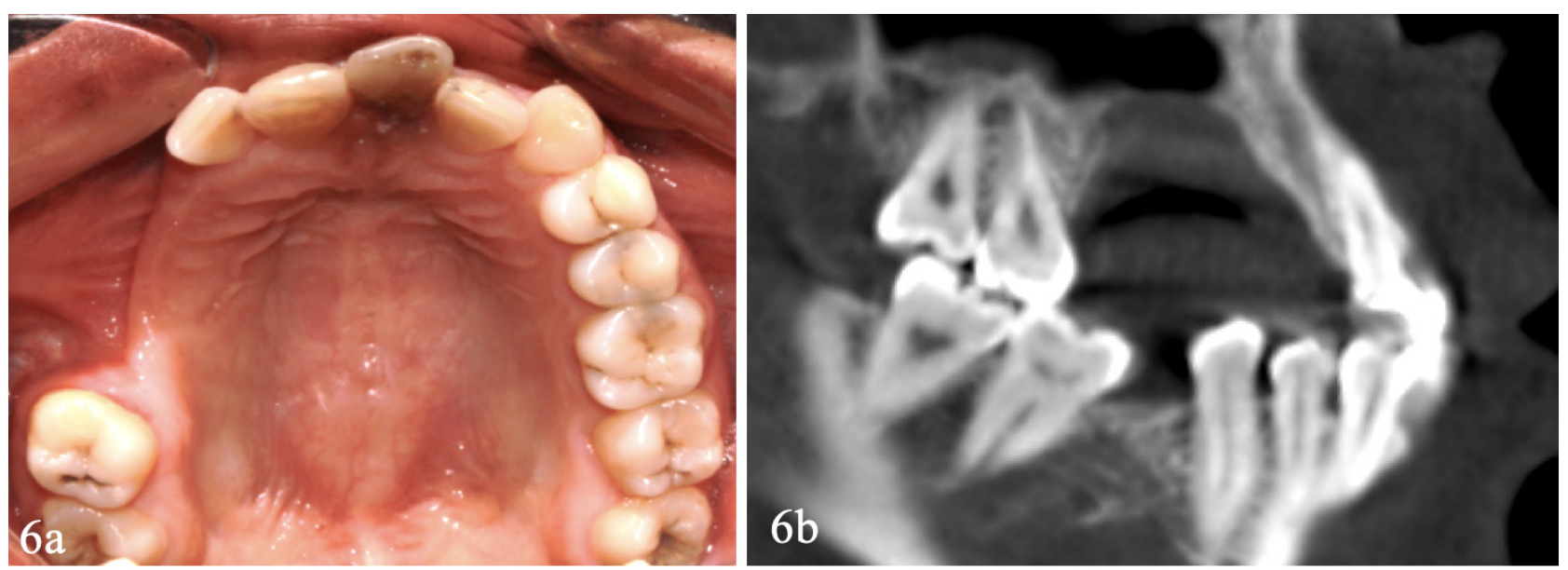

Figura 6. Control clínico y radiográfico después de 8 meses del tratamiento quirúrgico: 6a) Muestra la mucosa con características normales, 6b) Las paredes del hueso que limita al defecto creado por el procedimiento quirúrgico lucen normales.

\section{DISCUSIÓN}

El cementoblastoma es un tumor derivado del tejido odontogénico ectomesenquimal, está formado por proliferación de cementoblastos que producen cemento desorganizado, de allí que algunos autores usan los términos tejido mineralizado parecido al cemento $(3,4)$. Originalmente fue llamado cementoma verdadero y cementoblastoma benigno pero estos términos ya no se usan porque el primero causa confusión con otras entidades que presentan formación de cemento y la palabra benigno no es apropiada ya que no existe una contraparte maligna. Se considera que es la única neoplasia verdadera formadora de cemento, ya que, por ejemplo, en otras entidades como el fibroma cemento osificante además de cemento existe proliferación fibroblástica (3).

El cementoblastoma usualmente afecta una sola raíz (90\%), pero se han descrito casos desarrollados en varios dientes y en terceras molares impactadas tanto superiores como inferiores (5-7). Por otro lado, los cementoblastomas pueden por extensión comprometer el hueso de los dientes adyacentes como ocurrió en el caso reportado.

En las radiografías convencionales panorámica y periapical, clásicamente se lo describe como una masa radiopaca de forma redonda que se continua o está unido con la raíz de un diente rodeada por un espacio radiolúcido uniforme, sin embargo, también se han encontrado, aunque infrecuentemente producen imágenes radiolúcidas $(12,13)$. Sobre la base del reporte de Mohammadi et al., (11), se puede especular que el tumor se inicia como un área radiolúcida en estrecho contacto con la raíz de un diente, dando la impresión que se trata de una raíz de mayor longitud de lo normal, con el tiempo esta imagen radiolúcida evoluciona a una imagen radiopaca.

En la maxila a nivel de premolares y molares la imagen que se obtiene en las radiografías convencionales también corresponde a una masa radiopaca que se continúa con la raíz de un diente, pero su forma no aparece redonda ni se detecta el halo radiolúcido que envuelve al tumor, esto debido a la superposición de imágenes ocasionada por la conformación de las raíces, la curvatura del arco dental y la profundidad del paladar, como se ha demostrado en el presente caso.

El cementoblastoma presentado es interesante por varias razones: ocurrió en una zona rara, expandía simétricamente la corticales vestibular y palatina, cursaba con dolor y la radiografía panorámica y periapical no mostraban la imagen típica atribuida a esta lesión. Con la información obtenida y basados en la literatura (1-4,13-16), se plantearon los siguientes diagnósticos diferenciales: displasia cementaria periapical,osteoesclerosisidiopatica, hipercementosis, osteítis esclerosante focal, osteoma osteoide, osteoma central, osteoblastoma y osteosarcoma. Las dos últimas entidades exhiben radiopacidad y cursan con dolor, por otro lado, es importante señalar que un caso 
de osteosarcoma mandibular reportado en la literatura presentaba radiográficamente la imagen clásica de un cementoblastoma (14).

Si bien el estudio de TCHC aportó mayor información sobre las características imagenológicas, el hecho de encontrar en los cortes axiales y transaxiales (figura 3 (a y d)), filamentos hiperdensos en la periferie del tumor reforzó la necesidad de asegurar un diagnóstico correcto de la lesión antes de proceder a su remoción quirúrgica, lo que se logró con la toma de una biopsia, la cual fue informada como cementoblastoma.

El cementoblastoma es una neoplasia que tiene un crecimiento continuo que puede producir perforación de las corticales, su recurrencia ha sido estimada en $37,5 \%$ en un seguimiento de 5 años y en $11,8 \%$ en control de 2 años y medio respectivamente $(12,13)$, la recurrencia ocurre cuando queda parte del tumor después de un tratamiento quirúrgico incorrecto. En el caso presentado el tratamiento fue remoción en bloque que incluyó, además del tumor y la premolar, a las piezas 13 y 16 que estaban contiguas. El control a los 8 meses confirmó que el tratamiento fue correcto (figura 3 ( $a$ y b)), La ausencia de dientes y el defecto óseo alveolar resultados del tratamiento quirúrgico serán solucionados mediante una prótesis parcial removible.

El estudio de la pieza quirúrgica demostró que la lesión estaba fusionada con la cara mesial de la raíz de la premolar, extendiéndose tanto hacia vestibular como a lingual, histológicamente estaba rodeada completamente por una cápsula de tejido fibroso que se continua con el estroma del tumor (figura 5 (a)), por otro lado merece destacarse que el tipo de reabsorción que producía era por reemplazo o sustitución ya que no se encontraba actividad cementoclástica, sino que existía perforación del cemento normal y desaparición de la dentina por el crecimiento progresivo del cementoblastoma (figura 5 (b)).

Finalmente, se debe enfatizar que para establecer el diagnóstico definitivo de esta entidad es fundamental realizar un análisis integral de las características clínicas, hallazgos imagenológicos, obtenidos con TCHC y los resultados del estudio histopatológico de una biopsia representativa de la lesión. Por otro lado, siendo el tratamiento quirúrgico el indicado, este debe ser planificado considerando que a pesar de ser una lesión benigna existe un porcentaje importante de recurrencia señalada en la literatura científica sobre esta entidad llamada cementoblastoma.

\section{REFERENCIAS}

1. Dogra KS, Sharma A, Sharma N, Sharma A. Cementoblastoma a Rare Odontogenic Tumor-A Case Report and Differential Diagnosis. IJSS Case Report \& Reviews.2016; 3(3):1-3.

2. Bal Reddy P, Shyam NDVN, Shidar Reddy B, Kiran G, Prasad N. Cementoblastoma which was Associated with Maxillary First Premolar: An Anusual case report. J Clin Diagnostic Res.2012; 6(5):919-20.

3. Wright JM, Vered M. Update from the 4th Edition of the World Health Organization Classification of Head and Neck Tumours: Odontogenic and maxillofacial bone tumors. Head Neck Pathol. 2017; 11(1):68-77.

4. Neville BW, Damm DD, Allen CM, Bouquot JE. Oral and Maxillofacial Pathology. 3rd ed. Philadelphia: W.B. Sauders; 2009. p.655-56.

5. Kalburge JV, Kulkarni VM, Kini Y. Cementoblastoma affecting Mandibular First Molar-A Case Report. Pravara Med Rev. 2010; 2(4):33-7

6. Dadhich AS, Nilesh K. Cementoblastoma of posterior maxilla involving the maxillary sinus. Ann Maxillofac Surg. 2015; 5(1):127-9.

7. Cavalcante RC, Petinati MFP, Oliveira ER, et al. Benign Cementoblastoma Associated with an impacted third molar inside maxillary sinus. Case Rep Surg. 2018; 2018: 7148479.

8. Pathak J, Hosalkar RM, Sidana S, Swain N, Patel S. Benign cementoblastoma involving left deciduous first molar: A case report and review of literature. J Oral Maxillofac Pathol. 2019; 23: 422-8.

9. Nuvvula S, Manepalli S, Mohapatra A, Mallineni SK. Cementoblastoma Relating to Right Mandibular Second Primary Molar. Case Rep Dent. 2016; 2016:2319890.

10. Urs AB, Singh H, Rawat G, Mohanty S, Ghosh S. Cementoblastoma Solely Involving Maxillary Primary Teeth-A Rare Presentation. J Clin Pediatr Dent.2016; 40(2):147-51.

11. Mohammadi F, Aminishakib P, Niknami M, Razi Avarzamani A, Derakhshan S. Benign Cementoblastoma Involving Deciduous and Permanent Mandibular Molars: ACase Report. Iran J Med Sci. 2018; 43(6):6647.

12. Brannon RB, Fowler CB, Carpenter WM, Corio RL. Cementoblastoma: An innocuous neoplasm? A clinicopathologic study of 44 cases and review of the literature with especial emphasis on recurrence. Oral Surg Oral Med Oral Pathol Oral Radiol Endod. 2002; 93(3):311-20. 
13. Chrcanovic BR, Gomez RS. Cemetoblastoma: An updated analysis of 258 cases reported in the literature. J Cranio-Maxillofacial Surg. 2017; 45(10):1759-66.

14. Bilodeau E, Collins B, Costello B, Potluri A. Case Report: A pediatric case of cementoblastoma with histologic and radiographic features of osteoblastoma and osteosarcoma. Head and Neck Pathol.2010; 4(4):324-8.

15. Fang Z, Jin S, Zhang C, Wang L, He Y. Misdiagnosis of osteosarcoma as cementoblastoma from an atypical mandibular swelling: A case report. Oncol Lett. 2016; 11(6):3761-5.
16. Teixeira LR, Santos JL, Almeida LY, et al. Residual cementoblastoma: An unusual presentation of a rare odontogenic tumor. J Oral Maxillofac Surgery Med Pathol. 2018; 30(2):187-90

17. Brown JS, Shaw RJ. Reconstruction of the maxilla and midface: Introducing a new classification. Lancet Oncol.2010; 11(10):1001-8.

Recibido : 14-03-2021 Aceptado : 15-06-2021 